\title{
Downregulation of Extraembryonic Tension Controls Body Axis Formation in Avian Embryos
}

\author{
Daniele Kunz ${ }^{1,2}$, Anfu Wang ${ }^{1}$, Chon U Chan ${ }^{3}$, Robyn H. Pritchard ${ }^{4,5}$, Wenyu Wang ${ }^{5}$, Filomena Gallo ${ }^{6}$, \\ Charles R. Bradshaw ${ }^{1}$, Elisa Terenzani ${ }^{1}$, Karin H. Müller ${ }^{6}$, Yan Yan Shery Huang ${ }^{5}$, Fengzhu Xiong ${ }^{1,2,{ }^{*}}$ \\ ${ }^{1}$ Wellcome Trust / CRUK Gurdon Institute, University of Cambridge, Cambridge, UK \\ ${ }^{2}$ Department of Physiology, Development and Neuroscience, University of Cambridge, Cambridge, UK \\ ${ }^{3}$ Institute of Molecular and Cell Biology, A*STAR, Singapore \\ ${ }^{4}$ Department of Physics, University of Cambridge, Cambridge, UK \\ ${ }^{5}$ Department of Engineering, University of Cambridge, Cambridge, UK \\ ${ }^{6}$ Cambridge Advanced Imaging Centre, University of Cambridge, Cambridge, UK \\ *correspondence: fx220@cam.ac.uk
}

\begin{abstract}
Embryonic tissues undergoing shape change draw mechanical input from extraembryonic substrates. In avian eggs, the early blastoderm disk is under the tension of the vitelline membrane (VM). Here we report that chicken VM characteristically downregulates tension and stiffness to facilitate stage-specific embryo morphogenesis. While early relaxation of the VM impairs blastoderm expansion, maintaining VM tension in later stages resists the convergence of the posterior body causing stalled elongation, open neural tube, and axis rupture. Biochemical and structural analysis shows that VM weakening follows the reduction of its outer-layer glycoprotein fibers, which is caused by an increasing albumen $\mathrm{pH}$ due to $\mathrm{CO}_{2}$ release from the egg. Our results identify a previously unrecognized mechanism of body axis defects through mis-regulation of extraembryonic tissue tension.
\end{abstract}

Embryo development requires a regulated physical environment. Many animals evolved an embryo proper structurally supported by extraembryonic tissues which are further attached to the components of the egg. These connections allow transmission of materials, signals and forces between the embryo and the egg environment. As embryonic tissues change shape, they may be assisted or resisted physically by the connected structures. Measuring the mechanical dynamics of these structures and elucidating their regulatory mechanisms therefore are important for the understanding of developmental defects and the engineering of embryoids and organoids.

Early chicken embryos expand the disc-shaped blastoderm outwards prior to and during the first day after laying (D0, 0-24hrs) through extensive proliferation (Figure 1A; New, 1959; Eyal-Giladi and Kochav, 1976). In the next two days (D1-D2, 24-72hrs), the gastrula embryo converges prominently along the primitive streak and the forming body axis, reversing local tissue flow direction (Figure 1A; Hamburger and Hamilton, 1951; Rozbicki et al., 2015; Xiong et al., 2020; Saadaoui et al., 2020). During these times the outer rim of the blastoderm stays attached to a multi-layered protein structure enclosing the yolk: 
the vitelline membrane (VM), transmitting the tension on the $\mathrm{VM}$ to the embryonic tissues (New, 1959; Bellairs et al., 1969). Most ex ovo culture methods of the chicken embryo found that this attachment and a stretched VM are necessary for proper long-term development (New, 1955; Bellairs et al., 1967; Dugan et al., 1991; Chapman et al., 2001; Schmitz et al., 2016; Sydow et al., 2017), except when the blastoderm is supported on a gel substrate (Spratt, 1947) or when the tension can be supplied by inflation through water movement in a "Cornish pasty" culture (Connolly et al., 1995; Nagai et al., 2011). These studies point to an extraembryonic physical mechanism controlling early morphogenesis through the VM in ovo. To understand the mechanical dynamics of the VM, we first looked at how it maintains the yolk shape on a flat surface. The yolk index (height/diameter) reduces characteristically over incubation with or without embryos (Figures 1B-C; Moran 1936; Fromm, 1967). As the yolks do not show significant changes of weight, volume, or viscosity during these stages (Meuer and Egbers, 1990; Figure S1A), this shape change indicates that the VM becomes increasingly stretchable. We modelled the yolk shape as a liquid puddle and estimated the VM tension to be in the order of $0.1-1 \mathrm{~N} / \mathrm{m}$ between D0 and D2 (Figure S1B).

To measure VM tension, we extracted a piece of VM using a piece of windowed filter paper that sticks to the VM (Chapman et al., 2001) and then sandwiched it with a second piece of filter paper to maintain its endogenous stretched state. The sample is then connected to a weight on one end and vertically suspended on the other. Cutting the horizontal filter paper connections allows the weight to be supported by the VM alone so that tensions can be measured (Figures 1D-E, S2A). We found that, as development proceeds, VM sustains a reduced level of both native and maximum tension, the latter of which is consistent with pipette aspiration and texture analyzer experiments on the yolk to evaluate VM strength (Fromm and Matrone, 1962; Kirunda and McKee, 2000). To assess the viscoelastic properties of the VM, we used a force sensor to push perpendicularly on the filter paper VM samples to stretch the membrane and measured the rate of force increase (Figure S2B). We found that D0 VMs produce higher tension under the same deformation as compared to D2 VMs (Figure 1F). These results together show that VM reduces both tension and stiffness during early stages of development.

To test the role of VM mechanical changes in embryo morphogenesis, we first relaxed the VM prematurely on D0 by subtracting yolk in ovo causing a reduction of pressure on the VM. The blastoderm expansion decreased and embryos showed a shortened body axis (Figure 2A), resembling explanted cases where the VM is manually ruffled (Bellairs et al., 1967). Conversely, to prevent tension reduction entering D2, we sandwiched the normal ex ovo culture (Chapman et al., 2001) with an additional piece of windowed filter paper on D1.5 (36-40hrs, Figure S3A). This operation limits the ability of VM and extraembryonic tissues to move in the next 6hrs resulting in a stiffer VM similar to D1 rather than the actual stage of D2 (Figure 1F) and higher tissue tension as evidenced by faster wound opening in cutting experiments (Figures S3B-C). During this time, the sandwiched embryos start to show delay of convergence of the paraxial mesoderm and the neural tube in the posterior body axis (Figures 2B-D). Further prolonging this condition results in widening of the axis structures and cases of rupturing along the posterior neural midline or within the posterior paraxial mesoderm, areas known to produce forces that drive body axis morphogenesis (Xiong et al., 2020). In the anterior body, the stiffer 
somites and neural tubes are less affected but also show widening under prolonged sandwich culture. Notably, some embryos that avoid rupture go on to develop an open posterior neural tube with a normal tail fold (Figure 2E). To apply an increased VM tension directly, we used a double-ring culture device that pins down the VM over a liquid chamber which is connected to an external syringe enabling pressure control (Figures S3D-E; Sydow et al., 2017). Embryos show strain when the VM is inflated by media injection (Figures 2F-G), consistent with tension transmission between the VM and embryonic tissues (New, 1959). Over time, the posterior neural tube widens in inflated cultures, followed by similar posterior axis rupture as observed in sandwich experiments when higher tension is maintained. These data together show that the change of VM mechanics matches the stage-specific requirement of tension to facilitate normal tissue deformation including blastoderm expansion and body axis convergence.

To identify the mechanism of VM mechanical change, we stained for glycoproteins which are major structural components of the VM outer layer (Back et al., 1982). We observed significant reduction of fiber density in D2 VMs as compared to D0 (Figures 3A, S4A). Using electron microscopy cross-sections, we confirmed the reduction occurs in the outer layer (Figures 3D, S4B). This is consistent with an overall loss of proteins including glycoproteins on the VM during egg incubation (Figures S5A-B). We extracted ovomucin (a VM glycoprotein) and confirmed that its interaction with lysozyme (another VM component) weakens following $\mathrm{pH}$ increase in the albumen (Figure S5C, Cotterill \& Winter, 1954). Indeed, treating D0 VMs in pH 9.5 buffer greatly reduces glycoproteins within 12 hours (Figures 3B, S4A). Since it was known from early studies that the albumen $\mathrm{pH}$ increases from $\sim 7.5$ to $9-10$ from D0 to D2 of incubation due to the release of $\mathrm{CO}_{2}$ from pores in the eggshell (Fromm, 1967), we hypothesized that this chemical change causes VM weakening. To test this, we used mineral oil to block $\mathrm{CO}_{2}$ escape during egg incubation (Fromm, 1967). This perturbation stalled both the albumen pH increase and glycoprotein fiber reduction (Figures 3C, S5D). Importantly, VMs extracted from eggs that are fully oiled from D0 and D1 retain their respective stiffness by D2 (Figure 1F), indicating that VM weakening is stalled upon oil treatment. Strikingly, the embryos that develop in ovo under these conditions exhibit posterior body axis widening and rupture similar to those observed in ex ovo sandwich and inflation experiments (Figures 3D-F). While a fraction of eggs under oil treatments showed developmental failures and delays, the embryos that developed an intact body axis do not show other major defects except the body axis phenotypes, consistent with the low gas exchange requirement in these early stages (Sadler et al., 1954). The eggs that were fully oiled from D0 produced both the most ruptured embryos and the lowest albumen $\mathrm{pH}$. These data support the hypothesis that an increase in albumen $\mathrm{pH}$ mediated by $\mathrm{CO}_{2}$ escape causes the VM structural decay and the mechanical weakening necessary for the normal development of the posterior embryonic body.

Tissue morphogenesis does not occur in isolation but in connection to other tissues and the extraembryonic environment. To some extent, tissues can regulate force production against changes in the external resistance to promote robust deformation. However, such adaptation has its limits (Zhou et al., 2015). Our results show that during the early stages of chicken development tissue tension is regulated by biochemical modifications of the VM, assisting the tissues to achieve correct size and shape. This dynamic is likely 
conserved as inferred from similar yolk shape changes in other avian species (Imai et al., 1986; Damaziak et al., 2018). The strong early VM allows extraembryonic cells to generate sufficient tension at the blastoderm edge to drive tissue expansion (Bellairs et al., 1969), and the weak late VM allows body axis tissues to converge to the midline against low resistance. The changes of $\mathrm{CO}_{2}$ and albumen $\mathrm{pH}$ start after egg laying thereby ensuring that the timing of VM weakening aligns with the developmental progress of the embryo. In addition to the biochemical modification of the VM, water influx from the albumen to the yolk may contribute to tension regulation (Moran 1936). The capacity of tissue water transport is seen in chicken embryos in the Cornish pasty culture condition without the VM (Nagai et al., 2011) and may play a conserved role in size (and probably tension) control in mammalian early embryos (Chan et al., 2019). However, in the D0-D2 early-stage chicken embryos studied here, yolk size increase due to water transport is minimal (Meuer and Egbers, 1990) and does not appear to compensate for the tension reduction due to VM weakening. How the global mechanical changes of the VM interact with local stress anisotropy generated by patterned tissue behaviors (Saadaoui et al., 2020) remain to be characterized. The convergence of the body axis tissues is of particular interest given that it is an inward tissue flow and is the most strongly affected process under high tissue tension. The widened/open posterior neural tube in the more mildly perturbed embryos in our study mirrors certain neural tube defects (NTDs) that occur in 1/1000 human newborns (Wallingford et al., 2013; Fineman et al., 1986). Human embryos at these stages have a similar disc geometry (Molè et al., 2020) and may be under tension through connections with the trophectoderm. The tension dynamics and regulatory mechanisms for the human blastoderm remain unknown and can potentially be studied using in vitro cultures or embryoids derived from stem cells (Deglincerti et al., 2016; Moris et al., 2020). Our results and tools in the avian model enable further studies of genetic and cellular changes under tissue tension, which will shed light on the causes of body axis defects in this critical stage of human development.

Acknowledgement: The authors thank J. Rees, D. Shah, G. Wu, A. Sossick, P. Williamson, A. Downie, N. Smith, C. Jiggins, G. Sheng and members of the Xiong lab for reagents, technical assistance, and comments. This study is supported by a Wellcome Trust / Royal Society Sir Henry Dale Fellowship (215439/Z/19/Z) to F.X. Author Contributions: D.K. and F.X. designed and performed the experiments, analyzed the data and wrote the manuscript. A.W. contributed to data analysis. C.U.C, C.R.B. and E.T. contributed to the ring culture device; R.H.P., W.W. and Y.Y.S.H. contributed to the mechanical probe system. F.G. and K.H.M contributed to electron microscopy, which was performed using the facilities of the Cambridge Advanced Imaging Centre (CAIC). The authors declare no competing interest.

\section{References}

Back, J.F., Bain, J.M., Vadehra, D.V. and Burley, R.W., 1982. Proteins of the outer layer of the vitelline membrane of hen's eggs. Biochimica et Biophysica Acta (BBA)-Protein Structure and Molecular Enzymology, 705(1), pp.12-19.

Bellairs, R., Bromham, D.R. and Wylie, C.C., 1967. The influence of the area opaca on the development of the young chick embryo. Development, 17(1), pp.195-212. 
Bellairs, R., Boyde, A. and Heaysman, J.E., 1969. The relationship between the edge of the chick blastoderm and the vitelline membrane. Wilhelm Roux'Archiv für Entwicklungsmechanik der Organismen, 163(2), pp.113-121.

Chan, C.J., Costanzo, M., Ruiz-Herrero, T., Mönke, G., Petrie, R.J., Bergert, M., DizMunoz, A., Mahadevan, L. and Hiiragi, T., 2019. Hydraulic control of mammalian embryo size and cell fate. Nature, 571(7763), pp.112-116.

Chapman, S.C., Collignon, J., Schoenwolf, G.C. and Lumsden, A., 2001. Improved method for chick whole-embryo culture using a filter paper carrier. Developmental dynamics, 220(3), pp.284-289.

Connolly, D., McNaughton, L.A., Krumlauf, R. and Cooke, J., 1995. Improved in vitro development of the chick embryo using roller-tube culture. Trends in genetics, 11(7), pp.259-260.

Cotterill, O.J., Winter, A.R., 1954. The effect of $\mathrm{pH}$ on the lysozyme-ovomucin interaction. Poultry Science, 34, pp. 679-686.

Damaziak, K., Marzec, A., Kieliszek, M., Bucław, M., Michalczuk, M. and Niemiec, J., 2018. Comparative analysis of structure and strength of vitelline membrane and physical parameters of yolk of ostrich, emu, and greater rhea eggs. Poultry science, 97(3), pp.1032-1040.

Deglincerti, A., Croft, G.F., Pietila, L.N., Zernicka-Goetz, M., Siggia, E.D. and Brivanlou, A.H., 2016. Self-organization of the in vitro attached human embryo. Nature, 533(7602), pp.251-254.

Dugan Jr, J.D., Lawton, M.T., Glaser, B. and Brem, H., 1991. A new technique for explantation and in vitro cultivation of chicken embryos. The Anatomical Record, 229(1), pp.125-128.

Eyal-Giladi, H. and Kochav, S., 1976. From cleavage to primitive streak formation: a complementary normal table and a new look at the first stages of the development of the chick: I. General morphology. Developmental biology, 49(2), pp.321-337.

Fineman, R.M., Schoenwolf, G.C., Huff, M., Davis, P.L. and Prieur, D.J., 1986. Animal model: Causes of windowing-induced dysmorphogenesis (neural tube defects and early amnion deficit spectrum) in chicken embryos. American journal of medical genetics, 25(3), pp.489-505.

Fromm, D., 1967. Some physical and chemical changes in the vitelline membrane of the hen's egg during storage. Journal of Food Science, 32(1), pp.52-56.

Fromm, D. and Matrone, G., 1962. A rapid method for evaluating the strength of the vitelline membrane of the hen's egg yolk. Poultry Science, 41(5), pp.1516-1521.

Hamburger, V. and Hamilton, H.L., 1951. A series of normal stages in the development of the chick embryo. Journal of morphology, 88(1), pp.49-92.

Imai, C., Mowlah, A. and Saito, J., 1986. Storage stability of Japanese quail (Coturnix coturnix japonica) eggs at room temperature. Poultry Science, 65(3), pp.474-480. 
Kirunda, D.F.K. and McKee, S.R., 2000. Relating quality characteristics of aged eggs and fresh eggs to vitelline membrane strength as determined by a texture analyzer. Poultry Science, 79(8), pp.1189-1193.

Meuer, H.J. and Egbers, C., 1990. Changes in density and viscosity of chicken egg albumen and yolk during incubation. Journal of Experimental Zoology, 255(1), pp.16-21.

Molè, M.A., Weberling, A. and Zernicka-Goetz, M., 2020. Comparative analysis of human and mouse development: From zygote to pre-gastrulation. In Current Topics in Developmental Biology (Vol. 136, pp. 113-138). Academic Press.

Moran, T., 1936. Physics of the Hen's Egg: II. The Bursting Strength of the Vitelline Membrane. Journal of Experimental Biology, 13(1), pp.41-47.

Moris, N., Anlas, K., van den Brink, S.C., Alemany, A., Schröder, J., Ghimire, S., Balayo, T., van Oudenaarden, A. and Arias, A.M., 2020. An in vitro model of early anteroposterior organization during human development. Nature, pp.1-6.

Nagai, H., Lin, M.C. and Sheng, G., 2011. A modified cornish pasty method for ex ovo culture of the chick embryo. Genesis, 49(1), pp.46-52.

New, D.A.T., 1955. A new technique for the cultivation of the chick embryo in vitro. $J$ Embryol Exp Morphol 3: 320-331.

New, D.A.T., 1959. The adhesive properties and expansion of the chick blastoderm. Development, 7(2), pp.146-164.

Rozbicki, E., Chuai, M., Karjalainen, A.I., Song, F., Sang, H.M., Martin, R., Knölker, H.J., MacDonald, M.P. and Weijer, C.J., 2015. Myosin-II-mediated cell shape changes and cell intercalation contribute to primitive streak formation. Nature cell biology, 17(4), pp.397-408.

Saadaoui, M., Rocancourt, D., Roussel, J., Corson, F. and Gros, J., 2020. A tensile ring drives tissue flows to shape the gastrulating amniote embryo. Science, 367(6476), pp.453-458.

Sadler, W.W., Wilgus, H.S. and Buss, E.G., 1954. Incubation factors affecting hatchability of poultry eggs: 2 . Some effects of carbon dioxide upon morphogenesis. Poultry Science, 33(6), pp.1108-1115.

Spratt, N.T., 1947. A simple method for explanting and cultivating early chick embryos in vitro. Science, 106(2758), pp.452-452.

Sydow, H.G., Pieper, T., Viebahn, C. and Tsikolia, N., 2017. An Early Chick Embryo Culture Device for Extended Continuous Observation. In Avian and Reptilian Developmental Biology (pp. 309-317). Humana Press, New York, NY.

Schmitz, M., Nelemans, B. K., Smit, T. H., 2016. A Submerged Filter Paper Sandwich for Long-term Ex Ovo Time-lapse Imaging of Early Chick Embryos. J. Vis. Exp. (118), e54636, doi:10.3791/54636. 
Xiong, F., Ma, W., Bénazéraf, B., Mahadevan, L. and Pourquié, O., 2020. Mechanical coupling coordinates the co-elongation of axial and paraxial tissues in avian embryos. Developmental Cell, 55(3), pp.354-366.

Wallingford, J.B., Niswander, L.A., Shaw, G.M. and Finnell, R.H., 2013. The continuing challenge of understanding, preventing, and treating neural tube defects. Science, 339(6123).

Zhou, J., Pal, S., Maiti, S. and Davidson, L.A., 2015. Force production and mechanical accommodation during convergent extension. Development, 142(4), pp.692-701. 


\section{Figures}
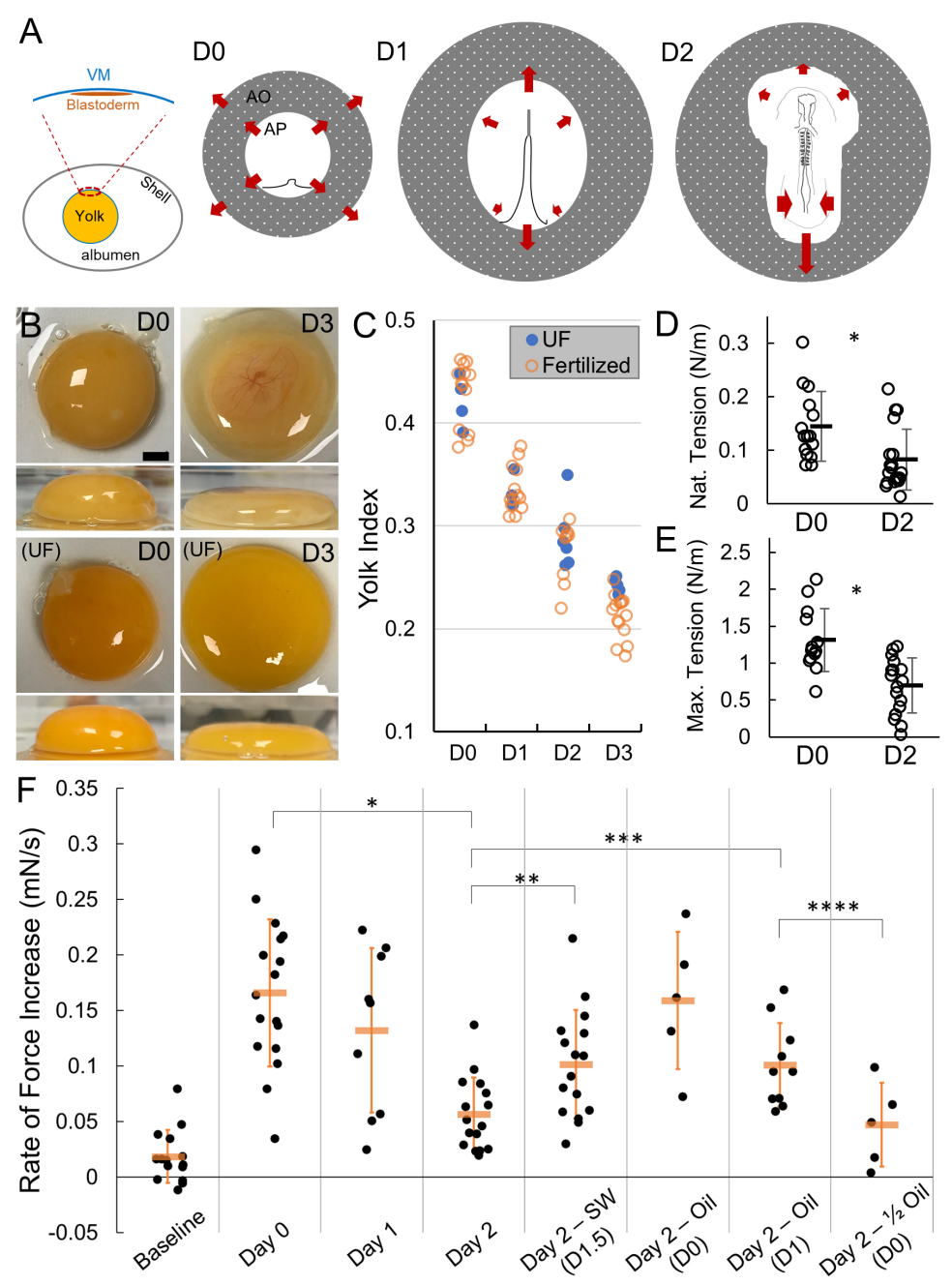

Figure 1. VM tension and strength decrease during early stages of development A. Diagram of early embryo tissue flow. Left panel shows the blastoderm in relation to the vitelline membrane (VM) and the egg. Right panels illustrate the early shape changes of the blastoderm including area opaca (AO) and area pellucida (AP). Each illustration (anterior to the top) shows the configuration of the blastoderm at approximately the beginning of the labeled incubation day (D0, D1 and D2). D0 involves blastoderm expansion and extension of the primitive streak. D1 and D2 involve continued regression of the streak, tissue convergence and body axis elongation that progress in an anterior to posterior order. Arrows indicate direction of tissue flow.

B. Yolk deformation on a flat surface. Top and side views. UF, unfertilized. Embryo is visible in the D3 image through the vasculature. Scale bar: $1 \mathrm{~mm}$.

C. Yolk index (height/diameter) over time. There is no significant difference between UF and fertilized ( $p>0.05$, t-tests).

D-E. Native (D) and maximum (E) tensions sustained by the VM. Bars indicate mean+/-SD. ${ }^{*} p<0.05$, t-tests. See also Figure S2A.

$\mathrm{F}$. VM stiffness measured by the rate of force increase under a stable rate of stretching.

Baseline samples do not contain VMs. Day labels indicate the amount of incubation time before the VM samples are measured. Texts following Day labels indicate perturbation type (after the 
dash) and perturbation start time (in "()"), respectively. For example, "SW (D1.5)" indicates filter sandwich (SW) perturbation started at D1.5. ${ }^{* * *},{ }^{* * *},{ }^{* * * *}$ indicate $p<0.05$, t-tests. See also Figure S2B.
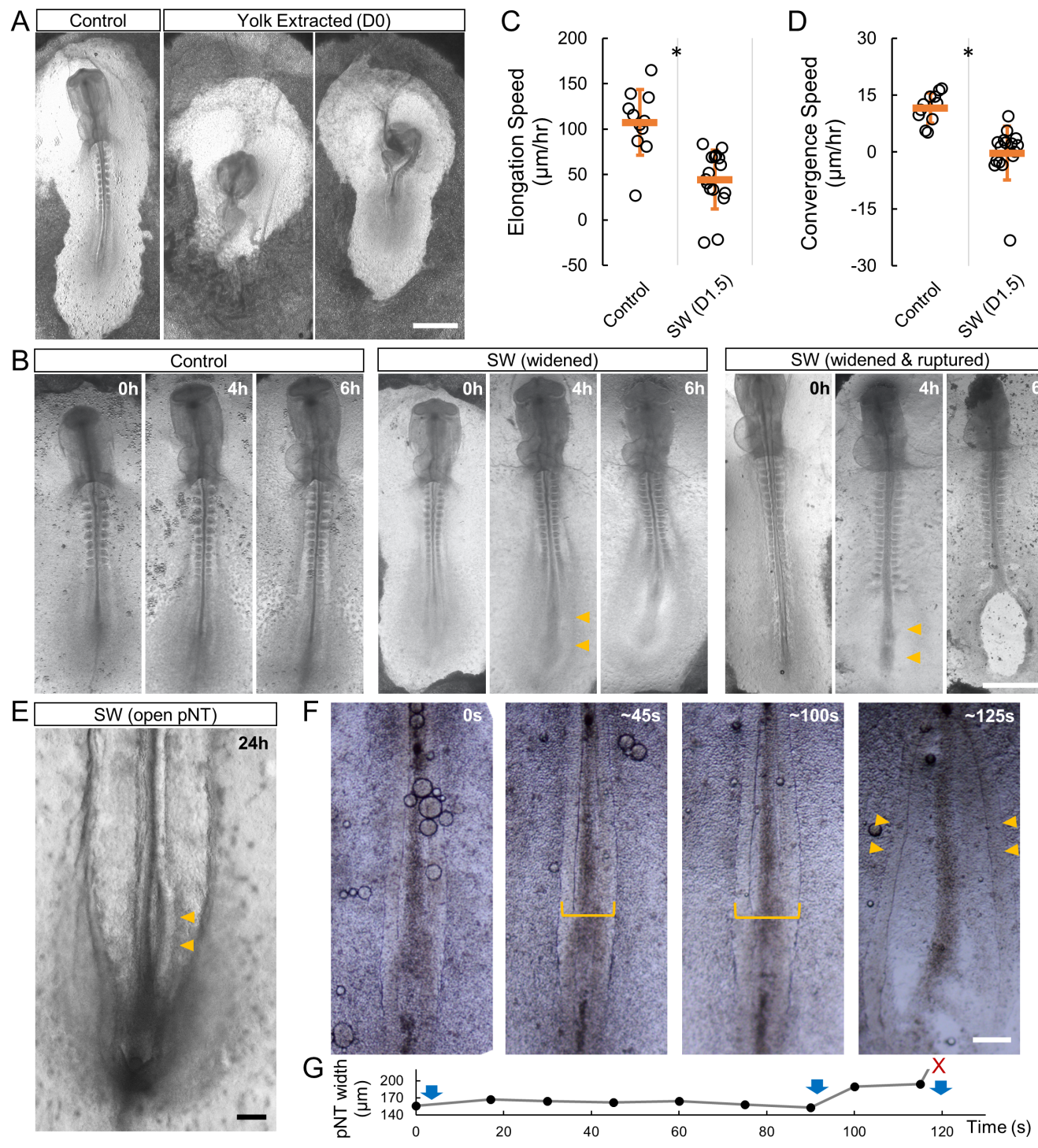

Figure 2. Changing VM tension causes stage-specific morphogenetic defects

A. Embryo phenotype (D1.5) following in ovo yolk extraction $(n=5) .2 .5 \mathrm{ml}$ yolk $(\sim 15 \% \mathrm{~V} / \mathrm{V})$ is removed by a syringe with a thin needle through an open window on the eggshell. Wrinkles of VM can be observed after yolk extraction. Eggs with no yolk-leaking are then resealed with tape and incubated. Scale bar: $1 \mathrm{~mm}$.

B. Embryo phenotypes (D1.5-D2) following filter sandwich (SW, see also Figure S3A). Time stamps indicate hours after the addition of the second filter. Arrowheads indicate the widening posterior body axis. A fraction of SW embryos ruptured under 6 hours $(n=11 / 37)$. No rupture was observed in controls $(n=0 / 20)$. Scale bar: $1 \mathrm{~mm}$.

C-D. Elongation (C) and convergence (D) speeds measured 6 hours after SW in embryos that do not rupture. Some embryos show a negative convergence speed i.e. widening and/or a negative elongation speed i.e. shortening. Bars indicate mean+/-SD. ${ }^{*} p<0.05$, t-tests. 
E. Open posterior neural tube (pNT) phenotype ( $n=3 / 10$, arrowheads) in extended SW culture at 24h post SW (D2.5). Scale bar: 100um.

F. Embryo phenotypes following inflation culture (see also Figures S3D-E). Time stamps indicate seconds after the start of live recording. Yellow brackets measure pNT width as shown in $(G)$. Arrowheads point to the NT tissue after rupture. Posterior body axis widening and/or rupture is observed in all samples tested $(n=10 / 10)$. Scale bar: $100 \mu \mathrm{m}$.

G. pNT width of the embryo in (E). Blue arrows indicate times of additional injections of medium to increase inflation and tension. Widening and rupture (red X) follow the injections.

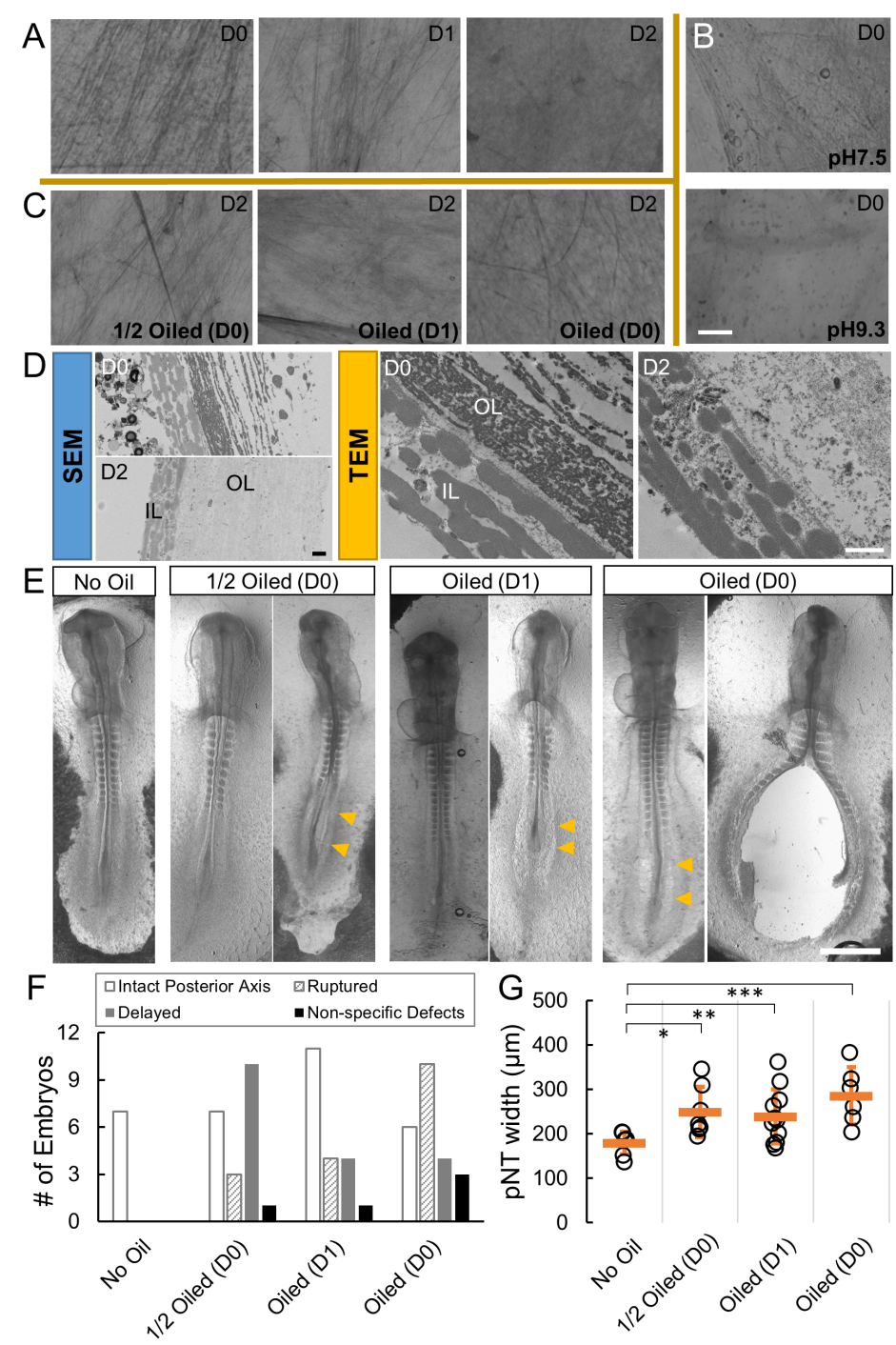

Figure 3. Biochemical changes of the egg environment control VM mechanics A-C. Glycoprotein fibers on the VM stained by the PAS (Periodic acid-Schiff) method. Top right label shows the incubation time when the VM is extracted. Bottom right label shows perturbations. Oil treatments start from the Day indicated in "()". pH buffer treatments last 12 hours. See Figure S4A for additional samples. Scale bar: $10 \mu \mathrm{m}$.

D. Scanning (SEM) and Transmission (TEM) electron microscope cross-sections of the VM. OL, outer layer; IL, inner layer. Scale bar, $1 \mu \mathrm{m}$. 
E. Embryo phenotypes following oil treatments. The embryos were extracted and imaged on D2. Images shown represent the variability of stages and phenotypes in oil treated embryos, summarized in (F). Arrowheads indicate widened posterior body axis tissues. Scale bar: $1 \mathrm{~mm}$. F. Summary of phenotypes following oil treatments. Aside from the ruptured, embryos with 7 or more pairs of somites are categorized as intact posterior axis, embryos with fewer than 7 pairs of somites are categorized as delayed. Embryos with no discernable axis are categorized in non-specific defects.

G. pNT width of the embryos with intact posterior axis. Bars indicate mean+/-SD. ${ }^{*},{ }^{* *},{ }^{* *} p<0.05$, t-tests. 


\section{Materials and Methods}

Avian eggs and incubation. Wild type fertilized chicken eggs are supplied by MedEgg Inc. Unfertilized eggs of similar sizes are obtained at Tesco. Eggs were kept in a monitored $15^{\circ} \mathrm{C}$ fridge for storage and $37.5^{\circ} \mathrm{C} \sim 45 \%$ humidity egg incubators (Brinsea) for incubation. The eggs are incubated within 2 weeks of receipt. No animal protocol is required for the embryonic stages studied under the UK Animals (Scientific Procedures) Act 1986 for incubation of chicken eggs under two weeks. To measure the yolk shape, eggs were opened into a petri dish and both thin and thick albumen was removed carefully with a Pasteur pipette. A top and a side photo of the yolk was then taken.

Scale measurement of VM tension. Incubated eggs were opened into petri dishes and the top center of the yolk area was cleaned gently to remove the albumen. A stripe of filter paper (Whatman) with two identically cut windows of $2 \mathrm{~cm}$-wide was folded in the middle to create a loop for a holding pipette mounted on a manual stage. The VM is first attached to one side of the filter paper and cut from the yolk. Then the other side is folded over to fix the VM piece in place. A clip is attached to the open end of the fold to stabilize the mounting as well as serving as the weight on the sample. The whole assembly is placed inside a digital balance scale (Fisher Scientific). The window has two small triangular cuts in the middle to allow easy release by cutting of the remaining filter paper supporting the weight. This also minimizes VM side deformation caused by the retraction at the cutting site due to tension. After cutting the VM suspends the weight alone. The scale reading of the weight reduces as the holder is raised. The tension is measured as the force difference over the width of the VM $(2 \mathrm{~cm})$. See also Figure S2A.

Mechanical probe system. A home-built system was used to measure VM mechanical properties. The system consists of a manual stage (Drummond) with a home-made sample holder, a self-illuminating miniature microscope camera (Kern), and a force sensor (UF1 Isometric, LCMSystems) on a motorized linear translation stage (Thorlabs) connected to a data acquisition system (DAQ, National instruments). See also Figure S2B. The holder uses screws and customprinted panels to fix the VM samples mounted on filter papers in place, allowing the system to accept samples prepared according to the embryo culture protocol (see the section below). The sensor data and the stage are controlled by a custom program created in LabView (National Instruments). During a measurement run, the VM sample is first mounted on the holder and the holder is then fixed on the manual stage which allows fine positioning of the VM sample to align perpendicularly to the force sensor. The sensor then starts logging the measurements. Next the sensor moves towards the VM sample at a constant speed of $0.1 \mathrm{~mm} / \mathrm{s}$ while the approach, contact and VM deformation are recorded from the side by the camera. The pushing phase lasts $40 \mathrm{~s}$ with contact of VM generally occurring between $5 \mathrm{~s}$ and $15 \mathrm{~s}$. After holding at maximum deformation for $10-15 \mathrm{~s}$, the probe is retracted at the same speed.

Embryo dish culture and filter sandwich. Embryos were staged following the Hamburger and Hamilton $(\mathrm{HH})$ table (Hamburger and Hamilton, 1951). Chicken embryos were extracted for ex ovo culture using the Early Chick (EC) culture system (Chapman et al., 2001). For D1.5 controls, the embryos are mostly between stages $9 \mathrm{HH}$ and $11 \mathrm{HH}$ (approximately 8-somite to 12-somite). To prepare the culture medium, the following formula was used: First, for $100 \mathrm{ml}$ of culture media, prepare Part A: $50 \mathrm{ml}$ Albumen (beaten for $15 \mathrm{~min}$ ) then supplemented with $0.8 \mathrm{ml} 20 \%$ Glucose and Part B: $0.3 \mathrm{~g}$ BactoAgar (Sigma) dissolved in $50 \mathrm{ml}$ water in a microwave then supplemented with $1.23 \mathrm{ml} 5 \mathrm{M} \mathrm{NaCl}$. Next, equilibrate part A and part B separately in a $55^{\circ} \mathrm{C}$ water bath. Finally, mix thoroughly and pipette $2 \mathrm{ml}$ each to $3.5 \mathrm{~cm}$ petri dishes before gelation. To prepare the filter paper mount for the embryo, $2 \mathrm{~cm} \times 2 \mathrm{~cm}$ pieces of filter paper (Whatman) were punched with two adjacent $0.5 \mathrm{~cm}$ holes. Eggs were opened into a petri dish. The thick albumen was swept aside 
gently near the top of the yolk around the embryo. The filter paper was then lowered with the embryo showing through the punched holes and pressed onto the VM. The VM was then cut around the filter paper to release the embryo. The samples were further rinsed in PBS to remove excess yolk. The cleaned embryos were placed ventral side up on pre-warmed culture dishes. The embryo cultures were maintained in a slide box lined with wet paper towels in the egg incubators $\left(37.5^{\circ} \mathrm{C} \sim 45 \%\right.$ humidity) except when snapshot imaging ( $<2 \mathrm{~min}$ per embryo) was performed. To perform the filter sandwich perturbation, an additional holed filter paper piece was attached to the ventral side of the embryo on the dish, and then wetted by PBS (see also Figure S3A). The embryos were imaged at different time points with a stereomicroscope (MZ10F, Leica).

Double-ring culture and inflation. The double-ring culture system was designed with modifications from a previous study (Sydow et al., 2017; see also Figure S3D). The base and cover are printed using an in-house 3D printer (Ultimaker S5) using BASF Ultrafuse PLA (Inno3D). The Engineering Profile $(0.15 \mathrm{~mm})$ was used with $20 \%$ infill and no supports. A spherical cover glass $(22 \mathrm{~mm}, V W R)$ was attached to the opening of the base ring by nail polish. The system is wetted with prewarmed culture medium (Part A only, see section above) before use. A $3 \mathrm{~cm} \mathrm{X}$ $3 \mathrm{~cm}$ square of filter paper with a $2 \mathrm{~cm}$ diameter hole on the center was used to extract the VM and embryo from the egg. The filter culture embryo was then rinsed in PBS to remove excess yolk. Cleaned VM and embryo were placed on top of the cover glass ventral side in the medium and clamped between the rings with screws. An externally attached syringe through a tunnel under the base ring allows culture medium to be supplied between the cover glass and the VM, inflating the clamped VM and achieving tension control. Inflation is controlled manually by slowly pressing the syringe to deliver $\sim 1 \mathrm{ml}$ of medium each time while observing the VM for rupture or leakage. The experiments are conducted on the stage of a stereomicroscope (10x objective, Kern Optics) and video recorded (see also Figure S3E). The running time of each experiment is usually short term (<10 minutes). With the exception of prewarmed medium, no other temperature control method was implemented.

In ovo perturbations. Yolk extractions: a small window $(\sim 1.5 \mathrm{~cm} \times 1.5 \mathrm{~cm})$ was opened in the eggshell on D0 eggs with a blade and scissors. Subsequently a fine pipette was used to puncture the VM and extract yolk from the egg $(\sim 2.5 \mathrm{ml})$. After yolk extraction the eggs were sealed with tape and incubated at $37.5^{\circ} \mathrm{C}$ for $38 \mathrm{~h}$ (D1.5). The embryos were extracted and imaged (Leica MZ10F). Samples that did not heal the VM resulting in yolk collapse would fail to develop embryos and are excluded from analysis. Oil treatment (adapted from Fromm, 1967): Eggs were dipped in mineral oil (Sigma) and wiped clean of excess oil and returned to the incubators. The oil stays on the eggshell pores stably over extended time (48h maximum in this study) as observed under bright light.

VM protein extraction and SDS-PAGE. 8 eggs per incubation time (0h; 24h; 36h; 48h; 54h) were opened into petri dishes and the VMs were cut open, removed from the dish and washed in PBS. The obtained VMs were dried in a SpeedVac. Proteins were extracted from the samples by using a buffer consisting of $50 \mathrm{mM}$ Tris- $\mathrm{HCl}(\mathrm{pH} 8.0), 10 \%$ glycerol, $2 \%$ sodium dodecyl sulfate (SDS), $25 \mathrm{mM}$ ethylenediaminetetraacetic acid, and protease inhibitor cocktail (Sigma-Aldrich). Samples were incubated overnight under constant stirring at room temperature. After this, the samples were centrifuged $\left(12000 \mathrm{~g}, 30 \mathrm{~min}, 4^{\circ} \mathrm{C}\right)$, the supernatant was collected, and the concentration of proteins was determined using the Lowry method. Sodium dodecyl sulfate polyacrylamide gel electrophoresis (SDS-PAGE) was carried out using $1.0 \mathrm{~mm}$ thickness of both running gel $(12 \%)$ and stacking gel $(5 \%)$ in the presence of SDS. The electrophoresis was run at $8 \mathrm{~V} / \mathrm{cm}$ in a Tris- $\mathrm{HCl}$ buffer system using a Vertical Biorad Mini attached to a Power Pac Basic electrophoresis apparatus. The samples were dissolved in $0.2 \mathrm{~mol} / \mathrm{L}$ Tris-HCl buffer $(\mathrm{pH} \mathrm{8.0)}$ containing SDS $(5 \mathrm{~g} / \mathrm{L})$ to a final concentration of $100 \mu \mathrm{g}$ of protein/lane. Protein solutions $(10 \mu \mathrm{l})$ 
along with a molecular weight ladder $(260 \mathrm{kD}$, Thermo Scientific) were loaded onto the gel and stained with colloidal Coomassie blue (Colloidal Blue Stain Kit, Invitrogen) or with Pierce ${ }^{\mathrm{TM}}$ Glycoprotein Staining Kit (Thermo Scientific) after separation.

Glycoprotein staining of the vitelline membrane. To examine the glycoprotein fibers on the VM, the samples were mounted on a cover slide and stained using Pierce ${ }^{\mathrm{TM}}$ Glycoprotein Saining Kit (Thermo Scientific), with PBS buffer wash to prevent drying. The stained VMs are examined microscopically at 100x (Kern Optics). To test the effect of $\mathrm{pH}$ changes on the VM glycoproteins, eggs without incubation (D0) were used to extract the VM. The samples were soaked in pH buffers of 7.5 and 9.3 . The buffers were made with $0.1 \mathrm{M}$ monopotassium phosphate and $0.05 \mathrm{M}$ borax (pH 7.9 to 9.5) and 0.05M phosphate buffer (Hawthorne, 1950). No adjustments were made for ionic strength. The VM samples were incubated overnight $(12 \mathrm{~h})$ at room temperature $\left(\sim 25^{\circ} \mathrm{C}\right)$ or in the incubator $\left(37.5^{\circ} \mathrm{C}\right)$.

Lysozyme-ovomucin interaction under different $\mathbf{p H}$. Ovomucin was prepared as described (Omana \& Wu, 2009). Albumen was diluted with 5 volumes of $0.1 \mathrm{~mol} / \mathrm{L} \mathrm{NaCl}$ solution and stirred gently for 30min using a magnetic stirrer. Subsequently, the $\mathrm{pH}$ of the mixture was adjusted to approximately 6 using $1 \mathrm{~mol} / \mathrm{L} \mathrm{HCl}$. The dispersion was kept overnight at $4^{\circ} \mathrm{C}$ and centrifuged at $15,000 \mathrm{~g}$ for $20 \mathrm{~min}$ at $4^{\circ} \mathrm{C}$. The precipitate was suspended in $0.5 \mathrm{~mol} / \mathrm{L} \mathrm{NaCl}$ solution and then kept at $4^{\circ} \mathrm{C}$ for $6 \mathrm{~h}$. After another centrifugation at $15,000 \mathrm{~g}$ for $20 \mathrm{~min}$ at $4^{\circ} \mathrm{C}$, the precipitate (containing ovomucin) was separated from the supernatant. The preparation was analyzed with SDS-PAGE to check for ovomucin. A few other protein bands were found to remain in the preparation but not expected to affect the following assay. The interaction between lysozyme and ovomucin was tested by turbidity as described (Cotterill and Winter, 1954). Tubes containing the ovomucin preparation were diluted $1: 2$ with $\mathrm{pH} 7.0$ to 9.3 buffers before the addition of purified lysozyme (2ug/ml, Sigma). The contents were then mixed and incubated at $37^{\circ} \mathrm{C}$ for 1 hour. The absorbance was measured at $450 \mathrm{~nm}$ by a microplate reader (Perkin Elmer 1420).

Sample preparation for electron microscopy. Eggs from different time incubation ( $0 \mathrm{~h} ; 48 \mathrm{~h} ; 54 \mathrm{~h})$ were used for VM extraction. Samples were fixed in a fixative (2\%glutaraldehyde/2\% formaldehyde in $0.05 \mathrm{M}$ sodium cacodylate buffer $\mathrm{pH} 7.4$ containing $2 \mathrm{mM}$ calcium chloride) overnight at $4^{\circ} \mathrm{C}$. After washing $5 \mathrm{x}$ with $0.05 \mathrm{M}$ sodium cacodylate buffer at $\mathrm{pH} 7.4$, samples were osmicated ( $1 \%$ osmium tetroxide, $1.5 \%$ potassium ferricyanide, $0.05 \mathrm{M}$ sodium cacodylate buffer at $\mathrm{pH}$ 7.4) for 3 days at $4^{\circ} \mathrm{C}$. After washing $5 \mathrm{x}$ in DIW (deionised water), samples were treated with $0.1 \%(\mathrm{w} / \mathrm{v})$ thiocarbohydrazide/DIW for 20 minutes at room temperature in the dark. After washing $5 x$ in DIW, samples were osmicated a second time for 1 hour at RT $(2 \%$ osmium tetroxide/DIW). After washing $5 x$ in DIW, samples were block-stained with uranyl acetate $(2 \%$ uranyl acetate in $0.05 \mathrm{M}$ maleate buffer at $\mathrm{pH} 5.5)$ for 3 days at $4^{\circ} \mathrm{C}$. Samples were washed $5 \mathrm{x}$ in DIW and then dehydrated in a graded series of ethanol (50\%/70\%/95\%/100\%/100\% dry), 100\% dry acetone and $100 \%$ dry acetonitrile, $3 x$ in each for at least $5 \mathrm{~min}$. Samples were infiltrated with a $50 / 50$ mixture of $100 \%$ dry acetonitrile/Quetol resin (without BDMA) overnight, followed by 3 days in 100\% Quetol (without BDMA). Then, the sample was infiltrated for 5 days in 100\% Quetol resin with BDMA, exchanging the resin each day. The Quetol resin mixture is: $12 \mathrm{~g}$ Quetol 651, $15.7 \mathrm{~g}$ NSA, $5.7 \mathrm{~g}$ MNA and $0.5 \mathrm{~g}$ BDMA (all from TAAB). Samples were placed in embedding moulds and cured at $60^{\circ} \mathrm{C}$ for 3 days.

TEM and SEM imaging. For TEM (transmission electron microscopy) imaging, sections (70nm thickness) were prepared in an ultramicrotome (Leica Ultracut) and placed on 300 mesh bare copper grids. Samples were viewed in a Tecnai G2 TEM (FEl/ThermoFisher) run at $200 \mathrm{keV}$ accelerating voltage using a $20 \mu \mathrm{m}$ objective aperture to improve contrast; images were acquired using an AMT digital camera. For SEM (scanning electron microscopy) imaging, thin sections 
were placed on Melinex (TAAB) plastic coverslips and allowed to air dry. Coverslips were mounted on aluminium SEM stubs using conductive carbon tabs (TAAB) and the edges of the slides were painted with conductive silver paint (TAAB). Then, samples were sputter coated with $30 \mathrm{~nm}$ carbon using a Quorum Q150 T E carbon coater. Samples were imaged in a Verios 460 scanning electron microscope (FEl/Thermofisher) at $4 \mathrm{keV}$ accelerating voltage and $0.2 \mathrm{nA}$ probe current in backscatter mode using the concentric backscatter detector (CBS) in field-free mode at a working distance of $3.5-4 \mathrm{~mm} ; 1536 \times 1024$ pixel resolution, $3 \mu \mathrm{s}$ dwell time, 4 line integrations. Stitched maps were acquired using FEI MAPS software using the default stitching profile and $10 \%$ image overlap.

Data analysis. Images and movies are processed by Fiji (NIH) and Powerpoint (Microsoft) for measurements. Scale bars are first set with control images with objects of known sizes. For elongation speed, the distance between a fixed somite pair (usually somite 3 or 4 ) and the posterior end of the body axis is taken. For posterior neural tube (pNT) width and convergence speed, the distance between the outer boundary of the neural tube walls is taken. For mechanical probe data, the raw trace (TDMS file, LabView) of each measurement containing the position of the probe and the force data over time was loaded into Matlab using custom code including a TDMS converter (Brad Humphreys, https://github.com/humphreysb/ConvertTDMS). The traces were examined alongside their corresponding live movies to exclude situations of early VM rupture and when probes hit the holder structure. The force curve of the admitted traces was first adjusted with their own baseline before probe movement started. The high frequency data points were then averaged to one value per second. The average rate of force increase of a 20 s duration over the approximately linear phase of force curve was used to measure the stiffness of the samples (See also Figure S2B). 


\section{Supplementary Information}

\section{Supplemental Figures}

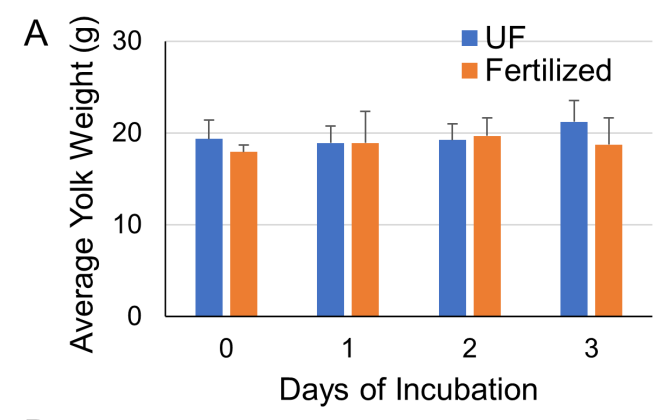

$\mathrm{B}$

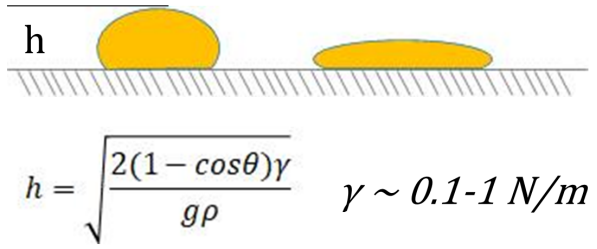

Figure S1. Physical changes of the yolk and the VM

A. Average yolk weight. Albumen was removed as much as possible for each yolk with a Pasteur pipette. $n=3-6$ for unfertilized eggs (UF); $n=8-14$ for fertilized eggs. Error bars show $+S D$. The weights are similar ( $p>0.05$, t-tests).

B. Simple droplet model of the yolk shape (De Gennes et al., 2013). $h$ (height) $1-2 \times 10^{-2} \mathrm{~m}$, $\rho$ (density) $\sim 1 \times 10^{3} \mathrm{~kg} / \mathrm{m}^{3}, g$ (gravitational acceleration $) \sim 10 \mathrm{~m} / \mathrm{s}^{2}, \theta$ (contact angle $)>90^{\circ}$.

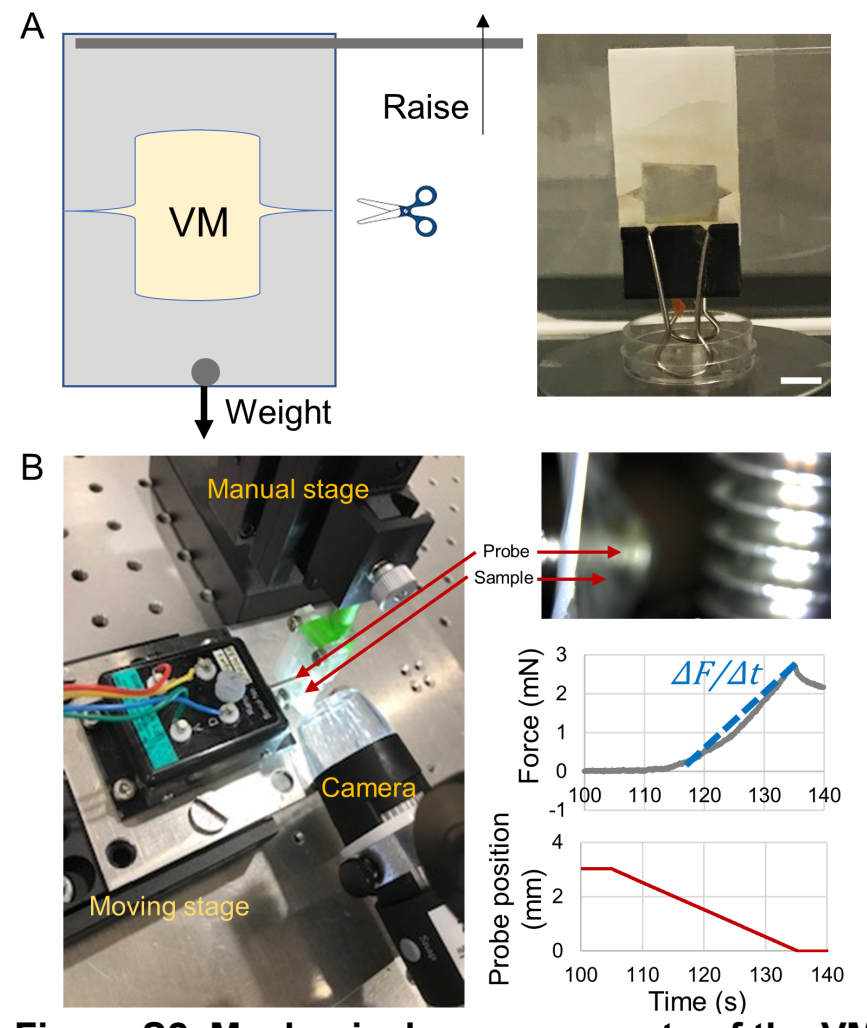

Figure S2. Mechanical measurements of the VM 
A. Tension measurement using a scale. Schematic on the left and an example on the right. The VM is sandwiched between two identical sides of a folded piece of windowed filter paper. A clip serving as the weight is attached on the bottom. Small contact points on both sides are designed in the window to minimize side deformation of the VM. After cutting these points the VM suspends the weight and raising the holder increases VM tension. Weight reading is recorded and analyzed in a live video. Scale bar, $1 \mathrm{~cm}$.

B. Stiffness measurement using a force probe. The left photo shows the components of the homemade system. Top right shows a measurement in progress from the camera view. The probe is seen to protrude and deform the VM towards the right side away from the filter mount. The plots show an example measurement sequence on a parafilm control. The probe moves at a stable speed and starts to detect a force when the sample starts to resist deformation. The average rate of increase $(\mathrm{mN} / \mathrm{s})$ of a $20 \mathrm{~s}$ duration over the approximately linear phase of force curve $(\Delta F / \Delta t$ in the plot) was used to measure $V M$ resistance.

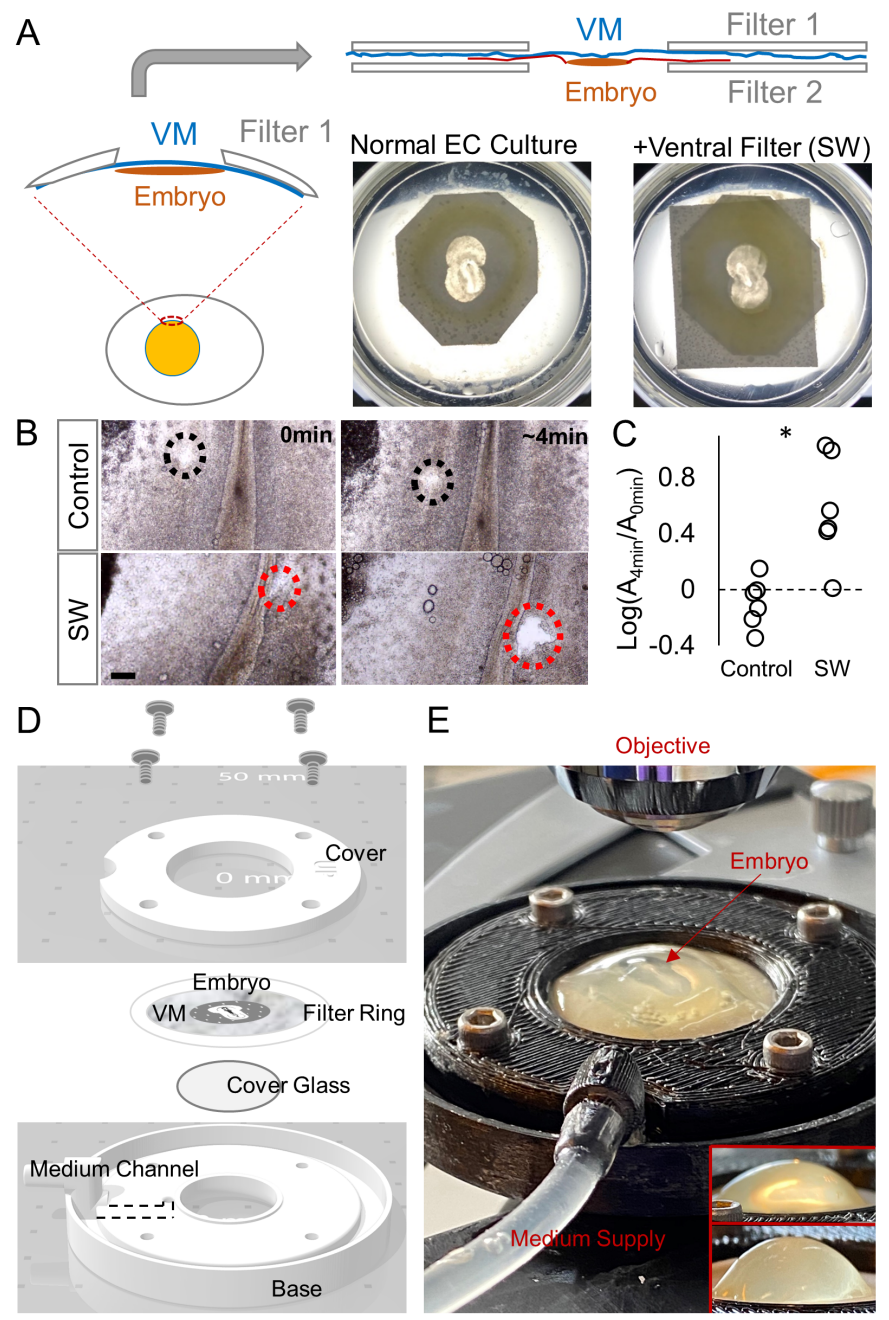

Figure S3. Mechanical perturbations of the VM

A. Filter sandwich (SW) to maintain tension of the VM. In the normal ex ovo culture protocol (Chapman et al., 2001), one piece of windowed filter paper (Filter 1) is attached to the VM and the embryo is cut out and laid down on the culture dish with ventral side up. By placing another filter (Filter 2) on the ventral side, the VM and the distal part of the extraembryonic tissue are sandwiched and fixed in place. Tissues in the window (including the embryo) can continue their movements. 
B. Wound opening after a needle cut on the embryos. Circles mark the wound site. Scale bar, $100 \mu \mathrm{m}$.

C. Wound size change. Areas at 0 min and approximately $4 \mathrm{~min}$ after cut are compared. Controls show slow opening or closing while SWs show expansion. ${ }^{*} p<0.05$ (t-test).

D-E. VM tension modulation with a double-ring inflation culture device. This system is a new design inspired by Sydow et al., 2017. The schematic (D) shows the components of the set-up. The photo (E) shows an experiment (Inserts show side views of different degrees of inflation, top is mild, bottom is strong). The cover and base (i.e. the double ring) are 3D printed. Embryo extracted (D1.5) on a filter ring is laid ventral side down on a cover glass which is glued to the opening of the base ring. Screws secure the seal of the sample allowing syringe controlled medium supply to inflate the sample. Samples with damage on the VM causing medium leaking are discarded.

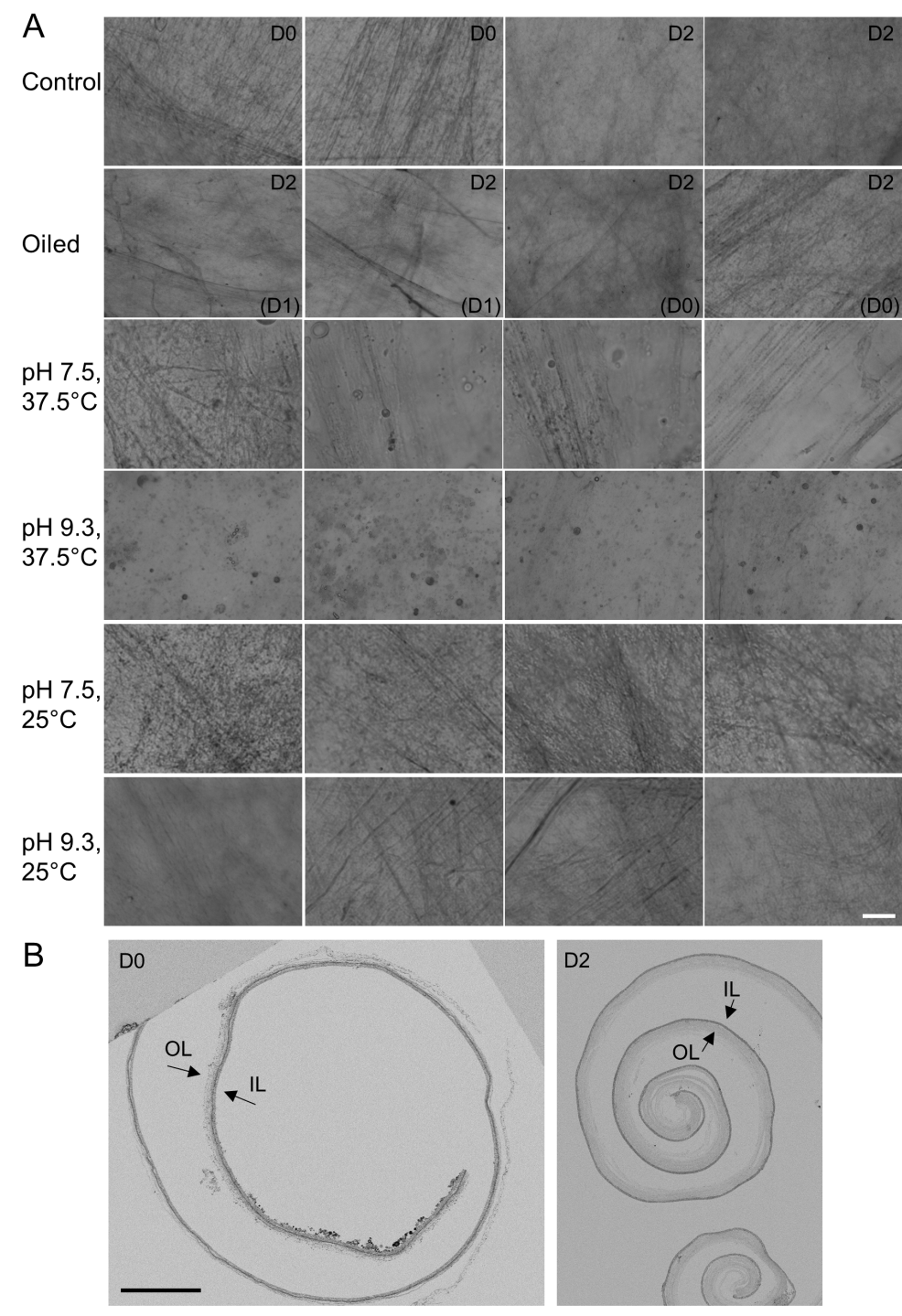

Figure S4. Structural changes of the VM

A. Glycoprotein fibers on the VM (these are additional examples related to Figure 3A). Top right label shows the Day when the VM is extracted and fixed for PAS staining. Bottom right label shows the Day when oil treatment starts. For the $\mathrm{pH}$ buffer treatment (12 hours) groups, all VMs are D0. Scale bar, $10 \mu \mathrm{m}$. 
B. Lower magnification SEM views of the VM cross-sections. OL, outer layer; IL, inner layer.

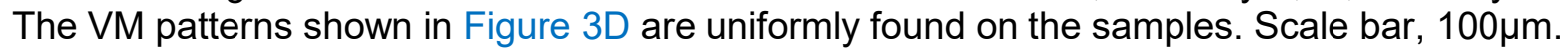
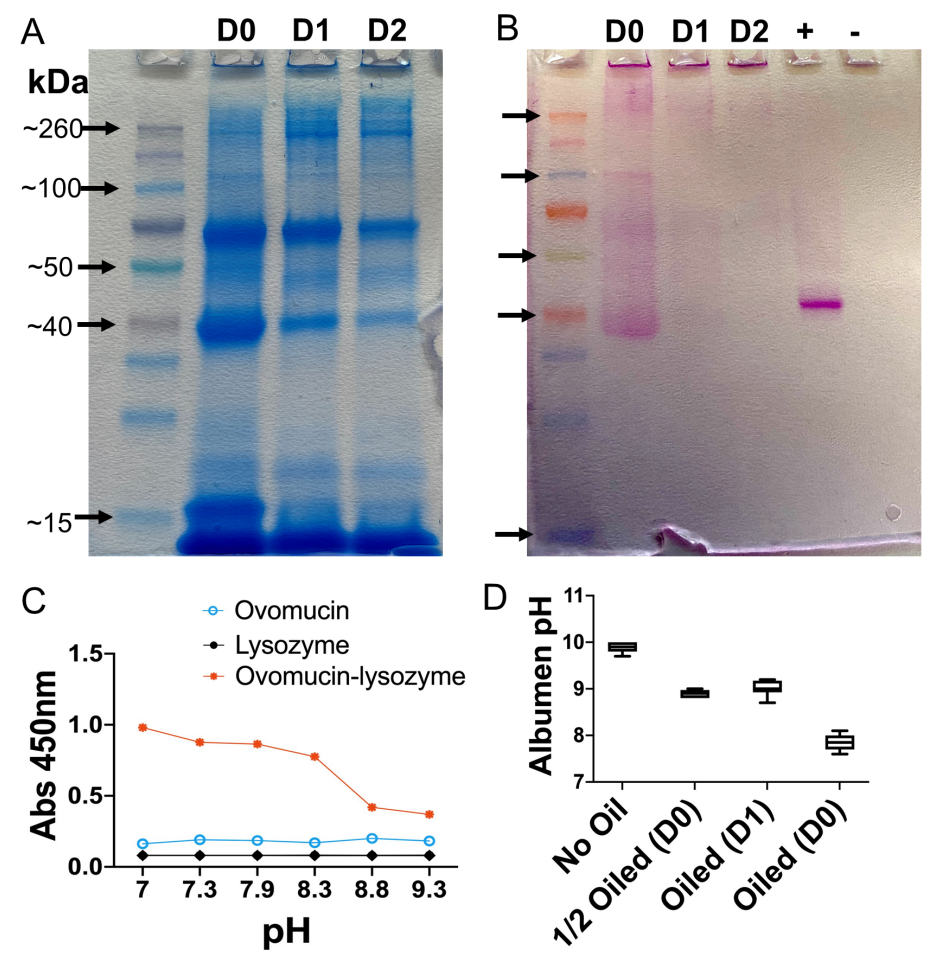

\section{Figure S5. Dynamics of VM proteins and albumen pH}

A-B. SDS gels of VM protein extraction stained by Coomassie blue and PAS for glycoproteins, respectively.

C. Interaction assay between Ovomucin and Lysozyme. Diluted preparations of two components fog the solution when mixed. This interaction is weakened by increasing $\mathrm{pH}$. D. Albumen $\mathrm{pH}$ under oil treatments ( $n=3-5$ for each group). Error bars are SD.

\section{Supplementary References}

De Gennes, P.G., Brochard-Wyart, F. and Quéré, D., 2013. Capillarity and wetting phenomena: drops, bubbles, pearls, waves. Springer Science \& Business Media. Chapter 2.2.

Omana, D.A. and Wu, J., 2009. A new method of separating ovomucin from egg white. Journal of agricultural and food chemistry, 57(9), pp.3596-3603.

Hawthorne, J.R., 1950. The action of egg white lysozyme on ovomucoid and ovomucin. Biochimica et biophysica acta, 6, pp.28-35. 